Peter Ullrich

\title{
Bounded Identity und Frameanpassung
}

\section{Article, Published version}

This version is available at http://nbn-resolving.de/urn:nbn:de:kobv:83-opus4-72777.

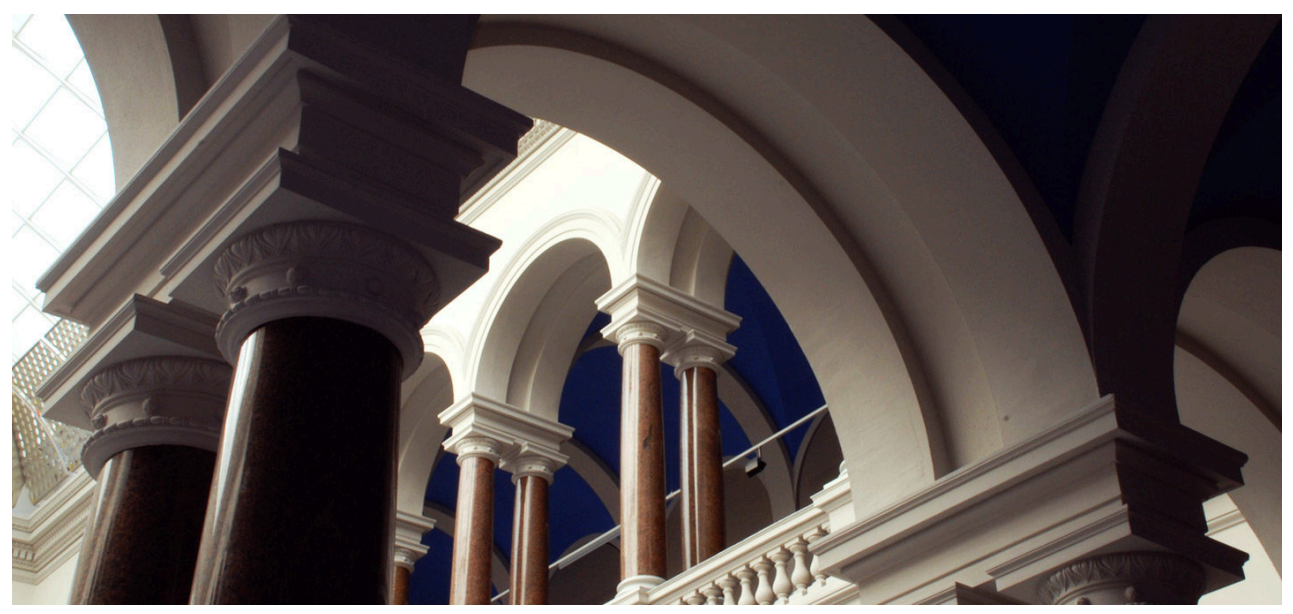

\section{Suggested Citation}

Ullrich, Peter: Bounded Identity und Frameanpassung. - In: Neue soziale Bewegungen :

Forschungsjournal. - ISSN: 0933-9361 (print), 2192-4848 (online). - 16 (2003), 2. - S. 127-132. 
in der Provinz Rio Grande do Sul und die brasilianische Präsidentschaftswahl. Die organisatorische Infrastruktur der Stadt, ohne die ein solches Großereignis nicht zu machen ist, war damit überfordert, zumal die PT die Provinzwahlen verloren hatte. Da durch den Präsidentenwechsel in Brasilia über $20.000 \mathrm{Be}$ amte ausgewechselt werden und Porto Alegre als Hochburg der PT eine erkleckliche Anzahl von Leuten in die Bundesregierung entsendet, fehlte es plötzlich auch an erfahrenem Personal. Aus all diesen Gründen gab es, anders als im Vorjahr, zahlreiche organisatorische Probleme, unter denen die kleinformatigen Veranstaltungsformen überdurchschnittlich litten. Wenn das Risiko der Stagnation bei der inhaltlichen und politischen Entwicklung des Sozialforums vermieden werden soll -, ein Problem, das auch beim europäischen Sozialforum in Florenz im vergangenen November bereits sichtbar wurde -, muss den kleinformatigen Veranstaltungen mehr Aufmerksamkeit gewidmet werden. Langfristig werden die Perspektiven der Bewegung davon abhängen, ob es gelingt, den Slogan, Eine andere Welt ist möglich ' in greifbaren, für größere Bevölkerungsgruppen attraktiven Alternativen mittlerer Reichweite zu konkretisieren.

Überlegenswert ist auch, ob nicht ein Zweijahresrhythmus des globalen Forums Sinn macht, alternierend mit den kontinentalen Foren, wie überhaupt über eine Entzerrung und Dezentralisierung nachgedacht werden muss. Letztlich wird das Gewicht der globalisierungskritischen Bewegung nur so groß sein, wie ihre Mobilisierungsfähigkeit im nationalstaatlichen Rahmen und vor Ort.

\section{Nächstes WSF in Indien}

Das nächste WSF wird in Indien stattfinden. Das ist eine Bereicherung. Eine Fixierung auf Porto Alegre führt auf Dauer zu Exklusivität, so sympathisch gerade uns Europäern Brasili- en auch ist. Sicher wird das Forum in Indien kleiner sein, aber das kann als Vorteil genutzt werden. Der kulturell völlig anders gelagerte Kontext in Indien wird andere Sichtweisen, andere Erfahrungen einführen. Es werden dabei sicher auch neue Probleme auftreten. Auf alle Fälle aber wird das WSF 2004 spannend.

Peter Wahl ist Mitarbeiter von WEED - Weltwirtschaft, Ökologie \& Entwicklung und Mitglied des Koordinierungskreises von Attac.

\section{FORSCHUNGSBERICHT}

\section{Bounded Identity und Frameanpassung}

\section{Die Mobilisierung nach Genua}

In der Literatur wird in bezug auf die sogenannten GlobalisierungsgegnerInnen oder GlobalisierungskritikerInnen gern von einem „Sammelsurium“ (Rucht 2001) von Bewegungen gesprochen oder von einem „bunte[n] Haufen“ mit „zum Teil ganz konträren Zielsetzungen“ (Leggewie 2000: 3), ,zu unspezifisch (...) um eine eigene Identität und Trennungslinien gegenüber den Gegnern zu markieren“ (Rucht 2001). Wie kann dann aber ein so großer und schlagkräftiger Protest wie in Genua entstehen? Die empirische Untersuchung ${ }^{1}$ der Proteste gegen den G8-Gipfel in Genua im Juli 2001 zeigt, dass es den beteiligten Akteuren durchaus gelingt, die Heterogenität der Ideologien und Zielvorstellungen nutzbringend zusammenzuführen und unter identitätsstiftenden Symbolen (Logos und Slogans), aber auch mit inhaltlichen Positionierungen (Flugblätter, Zeitungen) unterschiedlichste Akteure zu bündeln. ${ }^{2}$ Im Ergebnis kommt es zu mehr als nur dem schlichten Nebeneinanderher der „lose verknüpften, teils völlig unverbundenen Gruppen“ (Rucht 2001). 
Welche Strategien benutzen die Mobilisierungsakteure bei der Verwirklichung ihrer wichtigsten Aufgaben, der strukturellen (organisatorischen) und inhaltlichen (ideologischen) Integration dieses heterogenen Akteurspotenzials? Zur Beantwortung dieser Frage untersuchte ich die Struktur der Mobilisierung und die verwendeten Framingstrategien ${ }^{3}$, insbesondere sogenannte , interpretative Masterframes', d.h. repräsentative Arten, den Protest samt seiner Gründe, Ziele, Adressaten, Erfolgsaussichten und Selbstlegitimierung der Beteiligten auf einen begrifflichen Nenner zu bringen (Gerhards 1992: 315).

Die Ergebnisse zeigen jedoch, dass das notwendige $\mathrm{Ma}$ an empfundener inhaltlicher $\mathrm{Ge}$ meinsamkeit für erfolgreichen Protest nicht sehr groß ist. Der bunte Haufen hat in ideologischer Hinsicht nur eine , bounded identity'.

\section{Daten}

Grundlage der Studie waren v.a. qualitative Daten. In die Frameanalyse gingen Aufrufe, sich am Protest in Genau zu beteiligen, ein, darunter Flugblätter, Internetseiten und Parteibeschlüsse. Diese waren die Basis für eine Typologisierung des Spektrums in ideologischer und organisatorischer Sicht und für die Untersuchung ihrer anlassbezogenen interpretativen Masterframes und ihrer Globalisierungsvorstellungen. Des weiteren wurden viele Dokumente ausgewertet, aus denen sich die Struktur der Mobilisierung rekonstruieren ließ. Hauptquelle dafür waren Mailinglisten, mit deren Hilfe die Protestvorbereitungen koordiniert wurden, und SitzungsProtokolle von Vorbereitungstreffen der Mobilisierungsbündnisse, sowie Mobilisierungszeitungen, Presseerklärungen und Homepages. Hinzu kamen teilnehmende Beobachtung bei einem lokalen Genua-Mobilisierungsbündnis und Gespräche mit ,Experten * aus der Bewegung. Der Hauptfokus lag auf den Mesomobilisierungsakteuren, also Bündnissen, die mit ihrer vielfältigen und großen Unterstuitzerschaft jeweils eine gewisse Repräsentativität für Segmente der Bewegung beanspruchen können und deswegen hier vorgestellt werden sollen.

\section{Struktur der Mesomobilisierung}

Die Unterscheidung von Mikro- und Mesomobilisierung geht auf Gerhards und Rucht (1992) zurück. Die Mikromobilisierungsebene (vgl. Snow et al. 1986) bilden die einzelnen Gruppen und Personen, die in erster Linie versuchen, Individuen zu mobilisieren, und dabei an Mikromobilisierungskontexte (Freundschaftsnetzwerke u.ä.) anknüpfen. Die Mesomobilisierungsakteure und -gruppen sind auf der Ebene der Mobilisierungsbündnisse und anderer gruppenübergreifender Koordinationsformen angesiedelt. Sie mobilisieren in erster Linie Gruppen und andere Multiplikatoren. Diese Ebene lässt sich, wie später noch gezeigt werden soll, mehrfach untergliedern. Die bestehenden politischen Netzwerke zwischen Gruppen, Parteien und Bewegungen - immer mehr auch vermittelt durch Mailinglisten und Diskussionsforen im Internet - bilden den Mesomobilisierungskontext, an welchen die Mesomobilisierungsakteure anknüpfen. Die Akteure auf der Mesomobilisierungsebene leisten die wesentlichen Beiträge zur strukturellen und inhaltlichen Integration einer Protestkampagne. Sie drücken der Mobilisierung ihren Stempel auf. Diese Analyse gilt insbesondere für transnationale Proteste wie in Genua.

Auf drei Ebenen waren bei dieser Kampagne Mesomobilisierungsakteure wichtig: (a) glo$\mathrm{kal}$, (b) national und (c) regional/lokal.

a) Neben verschiedenen kommunistischen und anarchistischen internationalen Bündnissen spielte auf der übernationalen Ebene v.a. das Genoa Social Forum (GSF) eine wichtige Rolle, in einer Doppelfunktion sowohl 
als Hauptplattform für die internationale Koordination, aber auch verantwortlich für die konkrete Durchführung der Gegenaktivitäten und die Mobilisierung vor Ort (deswegen glokal). Es organisierte im Vorfeld zwei internationale Koordinierungstreffen. Ferner stellte es Protestinfrastruktur (Medien, Übernachtungsmöglichkeiten, Verpflegung etc.) bereit, versorgte Interessenten weltweit mit Informationen und veröffentlichte einen Aufruf, in dessen Zeichen die Proteste stattfanden. Das GSF organisierte auch den parallel zur G8-Tagung stattfindenden Gegenkongress. In thematischer Hinsicht dominierten dort Globalisierungskritik, Dritte Welt, Menschenrechte, Frieden und Umwelt ${ }^{4}$, doch die Palette der vertretenen Angebote war schier unbegrenzt.

b) Ein wesentlicher Pfeiler der Mobilisierungsstruktur war die nationale Ebene. In Deutschland war es das sogenannte ,Kasseler Bündnis;, welches alle überregional relevanten Vorbereitungen koordinierte. Dazu gehört die Abstimmung von Bussen, Verhaltensund Demonstrationsstrategien, Unterbringung und v.a. der Kontakt zum GSF und die Weiterleitung von Informationen über eine eigens eingerichtete Mailingliste und die vier bundesweiten Koordinierungstreffen. Auch das Kasseler Bündnis schrieb einen Aufruf, der von 25 Gruppen unterzeichnet wurde, die allerdings wiederum ein größeres Spektrum repräsentierten. ${ }^{5}$

c) Auf der regionalen und lokalen Ebene gab es ebensolche Bündnisse, zumindest in vielen größeren Städten (u.a. Berlin, Leipzig, München, Hannover, Bremen, Frankfurt), auch wieder mit Aufgabengebieten wie Transport, Unterbringung, Informationsvermittlung. Bei diesen Bündnissen kam es z.T. auch zu direkten Mobilisierungsaktivitäten auf der Mikroebene. Der Übergang zwischen Mikro- und Mesoebene ist hier also flieBend. Das Leipziger Bündnis beispielsweise organisierte als Bündnis eine Mobilisierungsveranstaltung, die einzelnen Gruppen führten jedoch auch unabhängig voneinander Aktionen und Veranstaltungen durch. Statt eines eigenen Aufrufs veröffentlichte das Bündnis einen Reader mit Aufrufen der Gruppen.

Die hier beschriebene Baumstruktur bildet die Basis der Protestorganisation. Sie ist aber nicht als hierarchisch zu misszuverstehen, sondern eingebettet in ein dezentrales Netzwerk, v.a. mit Überlappungen und vielen Beteiligten, die ,quer' zu diesen Strukturen agieren (bspw. Parteien und Bewegungsorganisationen, die auf allen drei Ebenen mitwirken oder lokale Gruppen, die beim internationalen, aber nicht beim nationalen Koordinationsgremium involviert waren usw.). In seiner segmentären Differenzierung orientiert es sich an bestehenden politischen Raumeinheiten, v.a. dem nationalstaatlichen Rahmen, durchbricht diese aber vielfach. Die hohe Bedeutung des nationalen Rahmens legt jedoch die Kennzeichnung der Proteste als international und nicht als transnational nahe. Auf allen Ebenen kommt eine ähnliche funktionsorientierte Aufgaben- (und Rollen-) Differenzierung hinzu: Es müssen organisatorische Aufgaben erledigt ${ }^{6}$ und es sollen Inhalte transportiert werden. Dazu im folgenden Abschnitt.

\section{Framingstrategien}

All diese Bündnisse mussten als zweite Voraussetzung für gelingende Protestmobilisierung auch gute Gründe liefern. Wie oben schon angedeutet, war aber das interessierte Spektrum hochgradig heterogen. Die Stichprobe bestand aus 42 Aufrufen von 20 Issue-Groups (darunter 6 heterogene Genua-Bündnisse, 4 Gewerkschaftsorganisationen, 3 Gruppen zu den Themen Globalisierung/Neoliberalismus, 2 mal MigrantInnen/ Antirassismus und 2 mal Dritte Welt, sowie je eine StudentInnen-, Umweltund kirchliche Gruppe) und 21 allgemeinpoli- 
tischen Gruppen/Parteien (darunter 14 radikale Linke und 7 sozialistische und kommunistische Organisationen). Diese Vielfalt betrifft alle Ebenen der Mobilisierung. Ein Beobachter von außen mag hier ein klassisch linkes Spektrum mit in erster Linie an das Wirtschaftssystem adressierten Forderungen erkennen. Innerhalb der Bündnisse erwiesen sich die Differenzen aber als ein ernstes Problem. Im Angesicht der gemeinsamen strategischen Interessen musste man deutsche Linksradikale, argentinische Umweltschützer, französische Bauern und baskische Nationalisten unter einen Hut bekommen.

Der GSF-Aufruf bspw. wurde von den verschiedensten Organisationen (fast 1200) von allen Kontinenten unterzeichnet. ${ }^{7}$ Das Dilemma, die große Vielfalt an Ideologien und Themenpräferenzen zu integrieren, löste das GSF mit Formulierungen, die erstens sehr allgemein blieben und zweitens bewusst die ,bewegte Vielheit' hervorkehrten. Die Problemdefinition war so keine Analyse im eigentlichen Sinne, sondern das Beschreiben von Phänomenen wie z.B. Armut. Dem wurden als Ausweg, und auch nur aufzählend, die vielfältigen Aktivitäten gegenübergestellt, ,mit denen wir uns engagieren für internationale Kooperation, Ökologie, BürgerInnen- und ArbeiterInnenrechte, Förderung ethischer und solidarischer Wirtschaftsmodelle (...)" usw. Die abschließende ,Forderung ' lautete: ,Eine andere Welt ist möglich ${ }^{8}{ }^{8}$ Das Wort Globalisierung kommt im übrigen in dem Aufruf nicht vor. Den Masterframe der Kritik nennt man am besten Ungerechte Weltordnung.

Auch das Kasseler Bündnis war ähnlich bunt zusammengesetzt. Es umging die Probleme, die sich durch die ideologische Heterogenität ergeben, durch einen ,Trick' (der im übrigen explizit beschlossen wurde): Berichtend wurden in seinem Aufruf, der überall in Deutsch- land verteilt wurde, die verschiedenen politischen Ansätze im Stil von ,Einige stellen sich...', , anderen geht es... oder ,und wieder andere...' nebeneinandergestellt, ohne dass klare Forderungen herauskamen. Die Forderungen beschränkten sich auf die Sätze ,Wir alle wollen, dass möglichst viele Menschen nach Genua fahren und dazu beitragen, dass die Aktionen dort zu einem Erfolg werden. Auf nach Genua!'. Globalisierungskritik kam nur als einer der aufgezählten Ansätze vor. Als Masterframe, der sowohl die (weitergehende) Kritik der radikalen als auch die der Reformer integriert (vgl. Pianta 2001: 190), fungierte die Kritik am Neoliberalismus.

Weniger heterogen als die beiden bisher beschriebenen Bündnisse, aber trotzdem auch von den Differenzen zwischen den beteiligten anarchistisch-herrschaftskritischen, antikapitalistischen und links-gewerkschaftlichen Gruppen geprägt war das Leipziger Bündnis. Noch expliziter als bei den anderen Bündnissen wird hier mit der Vielheit umgegangen. Es war den Gruppen einerseits nicht möglich, sich auf einen gemeinsamen Aufruf zu einigen, andererseits waren sie alle von einer zumindest strategischen, möglichst aber auch weiterreichenden Zusammenarbeit überzeugt. So entschied sich das Bündnis, eine Art Zeitung herauszugeben, die Aufrufe mehrerer der beteiligten Gruppen enthielt. Auf einer öffentlichen Diskussionsveranstaltung mit dem Ziel der Protestmobilisierung wurde den GruppenvertreterInnen ein kurzes Eingangsreferat ermöglicht, in welchem die gesehenen $\mathrm{Ge}$ meinsamkeiten beschrieben, aber auch $\mathrm{Ab}$ grenzungen vorgenommen wurden. Auch in diesem Bündnis spielte das Thema Globalisierung nur eine Nebenrolle. Im Zuge der langen Auseinandersetzungen um den Titel einigte sich das Bündnis auf den hier als Masterframe fungierenden Slogan, Gegen Ausbeutung und Unterdrückung . 
Wie hier leider nur am Rande ausgeführt werden kann, zeigt sich, dass der mediengängige Begriff ,Globalisierungsgegner" nur einen Teil der Bewegung wirklich bezeichnet, weil Kritik eher in den Masterframes ,Neoliberalismus", ,Kapitalismus“ oder einfach ,Ungerechtigkeit geübt wird. Das Konzept Globalität wurde mehrheitlich positiv besetzt wird, jedoch nicht im Hinblick auf die ökonomische Globalisierung, sondern auf die ,Globalisierung von Gerechtigkeit".

\section{Fazit}

Die untersuchten Fälle zeigen, dass ideologische Heterogenität ${ }^{9}$ kein Grund für das Nichtzustandekommen von Zusammenarbeit bzw. einer gemeinsamen, wenn auch begrenzten Protestidentität ist. Die Protestbewegung - und in ihr besonders die radikale Linke - erwies sich bei dieser Kampagne als hochgradig kompromissfähig und flexibel. Dies steht in einem krassen Gegensatz zu fast zeitgleich geführten harten Auseinandersetzungen (bspw. zwischen Attac und autonomen Gruppen) um Antisemitismus und den Nahostkonflikt, die wohl von stärker ausgeprägten, der Bewegung vorgelagerten Identitäten geprägt sind, in diesem Fall der nationalen Identität (Ullrich 2002b).

Die Gemeinsamkeit wird durch verschiedene Mechanismen hergestellt. Ein ganz wichtiger Mechanismus der Identitätsstiftung ist, was ich mit Frameanpassung bezeichnen möchte. In Fällen von Koordinierungsversuchen, in denen ideologische Kongruenz von an dem Thema G8-Gipfel Interessierten nicht bestand, kam es aus Gründen der inhaltlichen Integration zumindest zu Annäherungen. Allerdings argumentieren so entstehende Masterframes auf sehr allgemeinem Niveau und kehren oft Vielheit und Differenz als Stärke heraus. Nachfolgearbeiten müssten im Einzelfall untersuchen, $a b$ und wie beispielsweise Problemdeutungen von an Bündnissen beteiligten Gruppen durch die Zusammenarbeit ,nachhaltig' beeinflusst werden. Ebenso denkbar wäre auch, dass die inhaltlichen Annäherungen ausschließlich taktischer Art sind und somit keinen Einstellungswandel implizieren. Dies ist jedoch kein Gegenargument, denn massiv kommuniziert (in den Tausenden verteilter Flugblätter) und damit Basis des Massenprotestes wurden ja eben nicht die Einzelmeinungen ( $o b$ nun geändert oder nicht), sondern die Kompromisse.

Peter Ullrich promoviert z.Zt. zum Thema ,Politik und Identität. Zur Soziologie linker Identität in Deutschland‘. Email: angina8@web.de

\section{Anmerkungen}

${ }^{1}$ Der Artikel entsteht auf der Grundlage meiner Magisterarbeit. Diese ist online verfügbar und als Buch in Vorbereitung (Ullrich 2002a). Sämtliche hier nur kurzen Ausführungen sind dort en detail nachlesbar.

${ }^{2}$ In diesem Rahmen kann ich leider nicht auf die Symbole (z.B. die Weltkugel) und die Konstruktion einer gemeinsamen Geschichte (v.a. mit bezug auf die Zapatistenproteste, die Kampagne gegen das MAI und die Krawalle in Seattle) eingehen, sondern konzentriere mich auf die Analyse des Problemframings.

${ }^{3} \mathrm{Zu}$ den Strategien und Dimensionen des Framings vgl. Snow et al. (1986), Klandermans (1988), Gerhards (1992).

${ }^{4}$ Diese Rangfolge ergibt sich aus der quantitativen Themen-Analyse des Gegenkongresses.

${ }^{5}$ Man bedenke, dass allein Attac auch noch für seine Mitgliedsorganisationen BUND, ver.di (damals ÖTV und HBV), Jusos u.v.m. steht, und dass einige Gruppen die Erklärung im Wortlaut übernahmen, ohne sich auf die UnterzeichnerInnenliste setzen zu lassen.

${ }^{6}$ I.d.R. Vernetzung, Logistik (Transport, Unterkunft, Verpflegung), Finanzen, Vorgehen/ Taktik und Rechtliches.

7 Von den Unterzeichner-Organisationen kamen 1015 aus Italien, die anderen 170 verteil- 
ten sich wie folgt: $72 \%$ Europa, $12 \%$ Südamerika, $7 \%$ aus Nord- und Mittelamerika, 5\% aus Asien und 3\% aus Afrika. Etwas anders gestaltet sich die geografische Herkunft der ReferentInnen beim Gegenkongress. Von den im Programm eindeutig national zuzuordnenden kamen $44 \%$ aus Europa, 21\% aus Asien, $16 \%$ aus Südamerika, $11 \%$ aus Nordamerika und $7 \%$ aus Asien.

${ }^{8}$,Unsere Ziele', Aufruf des GSF, www.genoag8.org/doc-ger.htm [23.01.2002]

${ }^{9}$ Noch einmal sei betont: Da sich eigentlich nur schwer begründen lässt, was objektiv große bzw. kleine ideologische Heterogenität ist, sei die Selbstwahrnehmung der Akteure zum $\mathrm{Maß}$ genommen, die die Schwierigkeiten mit den Differenzen ja offen thematisieren.

\section{Literatur}

Gerhards, Jürgen 1992: Dimensionen und Strategien öffentlicher Diskurse. In: Journal für Sozialforschung, Heft 3/4, 307-318.

Gerhards, Jürgen/Rucht, Dieter 1992: Mesomobilization Contexts: Organizing and Framing in Two Protest Campaigns in West Germany. In: American Journal of Sociology 98 (3), 555 589.

Klandermans, Bert 1988: The Formation and Mobilization of Consensus, In: Klandermans, B./Kriesi, H./Tarrow, S. (Hg.): International Social Movement Research 1, Greenwich, London: JAI Press, 173-198.

Leggewie, Claus 2000: David gegen Goliath: Seattle und die Folgen. In: Aus Politik und Zeitgeschichte, B48, 3-4.

Pianta, Mario (2001): Parallel Summits of Global Civil Society. In: Anheier, H./Glasius, M./ Kaldor, M. (Hg.): Global Civil Society 2001, Oxford: Oxford University Press., 169-194.

Rucht, Dieter 2001: Zwischen Strukturlosigkeit und Strategiefähigkeit. Herausforderungen für die globalisierungskritischen Bewegungen. In: E+Z Entwicklung und Zusammenarbeit, 12, Dezember, 358-360, http://www.dse.de/zeit- schr/ez1201-6.htm [19.12.2001].

Snow, David A./Rochford jun., Burk/Worden, Steven K/Benford, Robert D. 1986: Frame Alignment Processes, Micromobilization and Movement Participation. In: American Sociological Review, 51, 464-481.

Ullrich, Peter 2002a: Gegner der Globalisierung? Organisation und Framing der Proteste gegen den G8-Gipfel in Genua, http://www. stud.uni - leipzig.de/ soz 96 jtv/ magisterarbeit.doc [11.03.2003], in Vorbereitung als: Peter Ullrich 2003, Gegner der Globalisierung?, Leipzig.

Ullrich, Peter 2002b: Projektionsfläche Naher Osten. PalästinenserInnen, Israelis und die deutsche Linke bei der Selbstzerfleischung, In: Kultursoziologie. Aspekte, Analysen, Argumente 2-02, 109-125.

\section{ANALYSE}

\section{Das Scheitern als Chance begreifen}

\section{Überforderung der Politik durch die Informationsfreiheit?}

Als Gerhard Schröder und Joschka Fischer am 20. Oktober 1998 mit viel Elan ihre Koalitionsvereinbarung begossen, hatten auch die Befürworter der Informationsfreiheit Grund zu feiern. Erstmals wurde das Ziel einer bundesdeutschen Regierung verankert, die Verwaltung des Bundes für die Bürger transparenter zu gestalten. In der Koalitionsvereinbarung hieß es: „Durch ein Informationsfreiheitsgesetz wollen wir unter Berücksichtigung des Datenschutzes den Bürgerinnen und Bürgern Informationszugangsrechte verschaffen. "Vier Jahre später tauchte erneut ein solcher Passus im Koalitionsvertrag auf. Rot-Grün hatte es in vier Jahren Regierungszeit nicht geschafft, ein Informationsfreiheitsgesetz (IFG) auf den Weg zu bringen - ein Armutszeugnis für die Bundes- 